\title{
Interaction of race and pathology for neuroendocrine tumors: Epidemiology, natural history, or racial disparity?
}

\author{
Danielle K. DePalo MD ${ }^{1}$ | Rachel M. Lee MD, MSPH ${ }^{1}$ (1) |
}

Alexandra G. Lopez-Aguiar MD, MS ${ }^{1}$ | Adriana C. Gamboa MD ${ }^{1}$ Flavio Rocha MD $^{2}$ |

George Poultsides $M^{3}$ | Mary Dillhoff $\mathrm{MD}^{4}$ | Ryan C. Fields $\mathrm{MD}^{5}$ |

Kamran Idrees $\mathrm{MD}^{6}$ | Hari Nathan $\mathrm{MD}^{7}$ | Daniel Abbott $\mathrm{MD}^{8}$ |

Shishir K. Maithel MD ${ }^{1} \mid$ Maria C. Russell MD ${ }^{1}$ | other members of the United States

Neuroendocrine Tumor Study Group

${ }^{1}$ Division of Surgical Oncology, Department of Surgery, Winship Cancer Institute, Emory University, Atlanta, Georgia

${ }^{2}$ Department of Surgery, Virginia Mason Medical Center, Seattle, Washington

${ }^{3}$ Department of Surgery, Stanford University Medical Center, Stanford, California

${ }^{4}$ Division of Surgical Oncology, The Ohio State University Comprehensive Cancer Center,

Columbus, Ohio

${ }^{5}$ Department of Surgery, Washington University School of Medicine, St Louis, Missouri

${ }^{6}$ Division of Surgical Oncology, Department of Surgery, Vanderbilt University Medical Center, Nashville, Tennessee

${ }^{7}$ Division of Hepatopancreatobiliary and Advanced Gastrointestinal Surgery, Department of Surgery, University of Michigan, Ann Arbor, Michigan

${ }^{8}$ Department of Surgery, University of Wisconsin School of Medicine and Public Health, Madison, Wisconsin

\section{Correspondence}

Maria C. Russell, MD, FACS, Associate Professor of Surgery Winship Cancer Institute, Division of Surgical Oncology, Department of Surgery, Emory University 1364 Clifton Road, Atlanta, GA 30322.

Email: maria.c.russell@emory.edu

\begin{abstract}
Background and Objectives: Although minority race has been associated with worse cancer outcomes, the interaction of race with pathologic variables and outcomes of patients with gastroenteropancreatic neuroendocrine tumors (GEP-NETs) is not known.

Methods: Patients from the US Neuroendocrine Study Group (2000-2016) undergoing curative-intent resection of GEP-NETs were included. Given few patients of other races, only Black and White patients were analyzed.

Results: A total of 1143 patients were included. Median age was 58 years, $49 \%$ were male, $14 \%$ Black, and $86 \%$ White. Black patients were more likely to be uninsured ( $7 \%$ vs $2 \%, P=.011$ ), and to have symptomatic bleeding ( $13 \%$ vs $7 \%, P=.009$ ), emergency surgery (7\% vs $3 \%, P=.006)$, and positive lymph nodes (LN) (47\% vs $36 \%, P=.021)$. However, Black patients had improved 5 -year recurrence-free survival (RFS) $(90 \%$ vs $80 \%, P=.008$ ). Quality of care was comparable between races, seen by similar LN yield, RO resections, postoperative complications, and need for reoperation/readmission (all $P>.05$ ). While both races were more likely to have pancreas-NETs, Black patients had more small bowel-NETs $(22 \%$ vs $13 \%, P<.001)$. LN positivity was prognostic for pancreas-NETs ( 5 -year RFS $67 \%$ vs $83 \%, P=.001$ ) but not for smallbowel NETs.

Conclusions: Black patients with GEP-NETs had more adverse characteristics and higher LN positivity. Despite this, Black patients have improved RFS. This may be attributed to the epidemiologic differences in the primary site of GEP-NETs and variable prognostic value of $\mathrm{LN}$-positive disease.
\end{abstract}

KEYWORDS

lymph node positivity, neuroendocrine tumors, pancreas, racial disparities, small bowel 


\section{1 | INTRODUCTION}

Although the majority are nonfunctional, neuroendocrine tumors are a heterogeneous group of cancers that can cause varied and nonspecific symptoms from the release of hormones. ${ }^{1,2}$ They are most commonly found in the gastrointestinal system, but can also be present in the pancreas, lungs, thyroid, ovaries, pituitary, and adrenal glands. ${ }^{1}$ Management includes surgical excision, which can be curative for localized disease, as well as surgical cytoreduction, radiotherapy, embolization, chemotherapy, and somatostatin analogues for patients with advanced disease. ${ }^{3}$ For the purpose of treatment and research, gastroenteropancreatic (GEP) neuroendocrine tumors are often grouped together. ${ }^{4}$ The most common locations of GEP neuroendocrine tumors are the small bowel and pancreas. ${ }^{1,5-7}$ While they both have similar management strategies, the prognosis of small bowel and pancreatic neuroendocrine tumors differs. ${ }^{1,8,9}$

There is a large body of research regarding racial disparities in cancer outcomes and treatments, but a relatively small amount specific to neuroendocrine tumors. Available data have shown that minority race and lower socioeconomic status are associated with not only more advanced stage at diagnosis, but also with variations in treatment received and differences in outcome for neuroendocrine tumors. ${ }^{1,5,10,11}$

Dasari et $\mathrm{al}^{5}$ found that Black patients with pancreatic neuroendocrine tumors had more advanced disease at diagnosis, limited utilization of surgery, and decreased disease-specific survival compared with patients of other races. Zhou et al ${ }^{11}$ likewise reported decreased overall and disease-specific survival for localized pancreatic neuroendocrine tumors in Black patients. ${ }^{11}$ In contrast, St Julien et $\mathrm{al}^{10}$ found that socioeconomic status and insurance coverage, but not race, were associated with variations in treatment and survival in patients with nonmetastatic pancreatic neuroendocrine tumors.

Most treatment and outcome disparities research for neuroendocrine tumors has been conducted by utilizing the Surveillance, Epidemiology, and End Results (SEER) database and National Cancer Database (NCDB), and largely focuses on pancreatic neuroendocrine tumors. ${ }^{5,10,11}$ Our aim was to investigate the interaction of race with clinicopathologic variables, disease presentation, treatment patterns, and recurrence-free survival (RFS) in GEP neuroendocrine tumors in a large multi-institutional database.

\section{2 | METHODS}

The United States Neuroendocrine Tumor Study Group (US NET-SG) is comprised of eight geographically diverse academic institutions: Emory University, University of Michigan, The Ohio State University, Stanford University, Vanderbilt University, Virginia Mason, Washington University, and University of Wisconsin. Institutional Review Board (IRB) approval was obtained at each study site before data collection. All patients who underwent resection for a neuroendocrine tumor from 2000 to 2016 were evaluated. A review of electronic medical records was conducted and pertinent baseline intraoperative, pathologic, and postoperative outcome data were collected. Staging was based on American Joint Committee on Cancer (AJCC) 7th edition guidelines. Data regarding neoadjuvant and adjuvant therapy, disease recurrence, and survival were also recorded.

Patients who underwent curative-intent resection for primary GEP neuroendocrine tumors were included. GEP neuroendocrine tumors were defined as originating in the ampulla, appendix, duodenum, pancreas, small bowel, or stomach. Patients with other concurrent malignancy, metastatic disease, or 30-day perioperative mortality were excluded. Due to few patients of other races, only non-Hispanic Black and non-Hispanic White patients were included in this analysis.

\section{1 | Statistical analysis}

SPSS version 25.0 Armonk New York Software (IBM Inc) was used for all statistical analyses. Descriptive and comparative analyses were used for the entire study cohort. Univariate and multivariable analysis of laboratory values, pathologic findings, and clinical outcomes were performed. $X^{2}$ analysis was used to compare categorical variables and the Student $t$ test or one-way analysis of variance was used for continuous variables, where indicated. Binary logistic and Cox regression analyses were used to compare preoperative variables with outcomes. Kaplan-Meier log rank tests were used for survival analysis. Statistical significance was predefined as $P<.05$.

\section{3 | RESULTS}

\subsection{Patient population and comparative data by race}

Of 2182 patients in the database, 1143 met inclusion criteria. Median age was 58 years and $49 \%$ were male. Median follow-up was 36 months. Fourteen percent $(n=157)$ of patients were Black and $86 \%(n=986)$ were White. Black patients were more likely to be female $(63.2 \%$ vs $48.8 \%, P=.003)$ and to be uninsured $(6.5 \%$ vs $2.4 \%$, $P=.011)$, to present with symptomatic gastrointestinal bleeding (12.7\% vs $6.5 \%, P=.009)$, and to require emergency surgery $(7.0 \%$ vs $2.5 \%, P=.006$ ) (Table 1 ). Black patients were more likely to present with tumors originating in the small bowel $(21.7 \%$ vs $12.8 \%, P<.001)$, though pancreas neuroendocrine tumors were the most common tumor type for both races (Table 1). Black patients were also more likely to have adverse pathologic characteristics at presentation including perineural invasion $(36.1 \%$ vs $25.2 \%, P=.046)$, positive lymph nodes $(47.3 \%$ vs $35.6 \%, P=.021)$, and presence of multifocal tumors $(13.0 \%$ vs $4.9 \%, P<.001)$.

The quality of surgical care received was similar between Black and White patients. There were no differences in lymph node yield at surgery (12 vs 12, $P=.804$ ), presence of positive margins after resection (12.2\% vs $14.6 \%, P=.498)$, incidence of postoperative 
TABLE 1 Baseline demographics and presentation by race

\begin{tabular}{|c|c|c|c|}
\hline Variable & White $(n=986)$ & Black $(n=157)$ & $P$ value \\
\hline Age $(y)$, mean $\pm S D$ & $56.7 \pm 13.7$ & $55.0 \pm 14.1$ & .159 \\
\hline Gender, n (\%) & & & .003 \\
\hline Female & $445(48.8)$ & $84(63.2)$ & \\
\hline Health insurance & & & .011 \\
\hline Uninsured & $23(2.4)$ & $10(6.5)$ & \\
\hline Presented with GI bleeding event & $64(6.5)$ & $20(12.7)$ & .009 \\
\hline Required emergency surgery & $25(2.5)$ & $11(7.0)$ & .006 \\
\hline Unknown primary at time of surgery & $38(3.9)$ & $16(10.2)$ & .001 \\
\hline NET found incidentally & $64(6.5)$ & $22(14.0)$ & $<.001$ \\
\hline Pancreas & $666(67.5)$ & $67(42.7)$ & \\
\hline Small bowel & $126(12.8)$ & $34(21.7)$ & \\
\hline Other GEP & $194(19.7)$ & $56(35.6)$ & \\
\hline Positive margin after resection & $142(14.6)$ & 19 (12.2) & .498 \\
\hline Lymphovascular invasion & $244(33.8)$ & $38(36.9)$ & .617 \\
\hline Perineural invasion & $162(25.5)$ & $30(36.1)$ & .046 \\
\hline Multifocal tumor & $42(4.9)$ & $20(13.0)$ & $<.001$ \\
\hline Lymph nodes retrieved (yes) & $271(35.6)$ & $52(47.3)$ & .021 \\
\hline Number $L N$ retrieved, mean $\pm S D$ & $11.7 \pm 9.4$ & $12.0 \pm 8.5$ & .804 \\
\hline Readmission & $185(18.9)$ & $30(19.1)$ & 1.000 \\
\hline Recurrence & $143(15.0)$ & $12(7.6)$ & .019 \\
\hline
\end{tabular}

Abbreviations: GI, gastrointestinal, NET, neuroendocrine; SD, standard deviation.

Bold values denote $P<.05$.

complications $(39.7 \%$ vs $47.5 \%, P=.086)$, need for reoperation $(4.1 \%$ vs $4.4 \%, P=1.00$ ), or readmission ( $19.1 \%$ vs $18.9 \%, P=1.00)$ in Black compared with White patients.

\subsection{Comparative data by primary tumor location}

Patients with small bowel primaries were older $(59.7 \pm 12.3$ vs $56.1 \pm 13.9$ years, $P=.002)$ and more likely to be Black $(21.3 \%$ vs $9.1 \%, P<.001$ ) than patients with pancreas primary tumors (Table 2 ). Patients with small bowel primaries were also more likely to have adverse pathologic characteristics with increased rates of lymphovascular invasion $(66.7 \%$ vs $28.5 \%, P<.001)$, perineural invasion (52.0\% vs $22.2 \%, P<.001$ ) and positive lymph nodes ( $82.1 \%$ vs $24.5 \%$, $P<.001)$. Patients with pancreatic primaries were more likely to have postoperative complications $(54.9 \%$ vs $36.5 \%, P<.001)$, in particular anastomotic leak $(24.5 \%$ vs $2.1 \%, P<.001)$. The rates of reoperation, readmission, and recurrence between the two sites were similar.

\section{3 | Recurrence-free survival}

When considering the entire cohort, Black patients had improved 5-year RFS compared with White patients $(89.9 \%$ vs $79.5 \%, P=.008$ ) (Figure 1). When examining small bowel neuroendocrine tumor RFS by race and stratifying by lymph node status, there was no significant difference in 5-year RFS in Black and White races (lymph node negative $P=.540$, lymph node positive $P=.388$; Figure $2 A$ ). However, for pancreatic neuroendocrine tumors there was a significant difference in 5 -year RFS in lymph node negative tumors by Black and White races 
TABLE 2 Comparative data by primary tumor location

\begin{tabular}{|c|c|c|c|}
\hline Variable & $\begin{array}{l}\text { Pancreas } \\
(n=733)\end{array}$ & $\begin{array}{l}\text { Small bowel } \\
(n=160)\end{array}$ & $P$ value \\
\hline Age & $56.1 \pm 13.9$ & $59.7 \pm 12.3$ & .002 \\
\hline Gender & & & .953 \\
\hline Male & $371(50.6)$ & $82(51.3)$ & \\
\hline Female & $362(49.4)$ & $78(48.8)$ & \\
\hline Race & & & $<.001$ \\
\hline White & $666(90.9)$ & $126(78.8)$ & \\
\hline Black & $67(9.1)$ & $34(21.3)$ & \\
\hline $\begin{array}{l}\text { Positive margin after } \\
\text { resection }\end{array}$ & $110(15.1)$ & $14(8.9)$ & .053 \\
\hline Lymphovascular invasion & $171(28.5)$ & $78(66.7)$ & $<.001$ \\
\hline Perineural invasion & $121(22.2)$ & $52(52.0)$ & $<.001$ \\
\hline $\begin{array}{l}\text { Lymph nodes retrieved } \\
\text { (yes) }\end{array}$ & $615(84.4)$ & $142(89.9)$ & .099 \\
\hline Positive lymph nodes & $151(24.5)$ & $119(82.1)$ & $<.001$ \\
\hline $\begin{array}{l}\text { Any postoperative } \\
\text { complication }\end{array}$ & $402(54.9)$ & $58(36.5)$ & $<.001$ \\
\hline $\begin{array}{l}\text { Multiple postoperative } \\
\text { complications }\end{array}$ & $169(42.1)$ & $22(37.9)$ & .641 \\
\hline Anastomotic leak & $171(24.5)$ & $3(2.1)$ & $<.001$ \\
\hline Reoperation & $25(3.6)$ & $10(6.9)$ & .111 \\
\hline Readmission & $169(23.1)$ & $27(17.1)$ & .121 \\
\hline Recurrence & $94(13.0)$ & $26(17.7)$ & .168 \\
\hline \multicolumn{4}{|l|}{$\begin{array}{l}\text { 5-y Recurrence-free } \\
\text { survival }\end{array}$} \\
\hline Entire cohort & $81.1 \%$ & $81.9 \%$ & .890 \\
\hline White patients only & $81.1 \%$ & $79.2 \%$ & .341 \\
\hline Black patients only & $81.1 \%$ & $91.1 \%$ & .160 \\
\hline
\end{tabular}

Abbreviation: SD, standard deviation.
(100\% vs $81.7 \% ; P=.013$; Figure $2 \mathrm{~B}$ ), while 5 -year RFS remained comparable by race in lymph node-positive disease $(68.6 \%$ vs $67.0 \%$; $P=.960$; Figure 2B). When assessing the prognostic value of lymph node-positive disease, regardless of race, on Cox regression analysis, lymph node positivity was not predictive of RFS in small bowel neuroendocrine tumors (Table 3a). Lymph node positivity, however, was associated with decreased RFS in pancreatic neuroendocrine tumors (HR, 1.99; 95\% Cl, 1.30-3.06; $P=.002$; Table 3b).

\section{4 | DISCUSSION}

Black patients undergoing surgery for curative intent resection of GEPNETs presented with more advanced pathologic characteristics compared with White patients. Although there may be delays in Black patients seeking or reaching care, as evidenced by these worse pathologic variables at presentation, they received similar quality of care compared with White patients at these tertiary referral centers that comprise the US NET-SG. Despite having more advanced disease at presentation, Black patients had improved RFS compared with White patients, suggesting that similar quality of surgical care is being offered to both races.

This is contradictory to much of the available literature regarding the impact of race on outcomes. ${ }^{5,11,12}$ However, it is important to note that our study population is limited to patients receiving curative-intent surgical care at select academic high-volume institutions. This may in part account for racial differences in severity of disease at presentation since care was still accessed early enough to be amenable for curative-intent resection. Given our study cohort of only patients who underwent curative-intent resection, we cannot assess any potential racial disparities in the utilization of high-volume academic centers. ${ }^{12}$

Others in the literature that have primarily focused on race associated outcomes of pancreatic neuroendocrine tumors using SEER

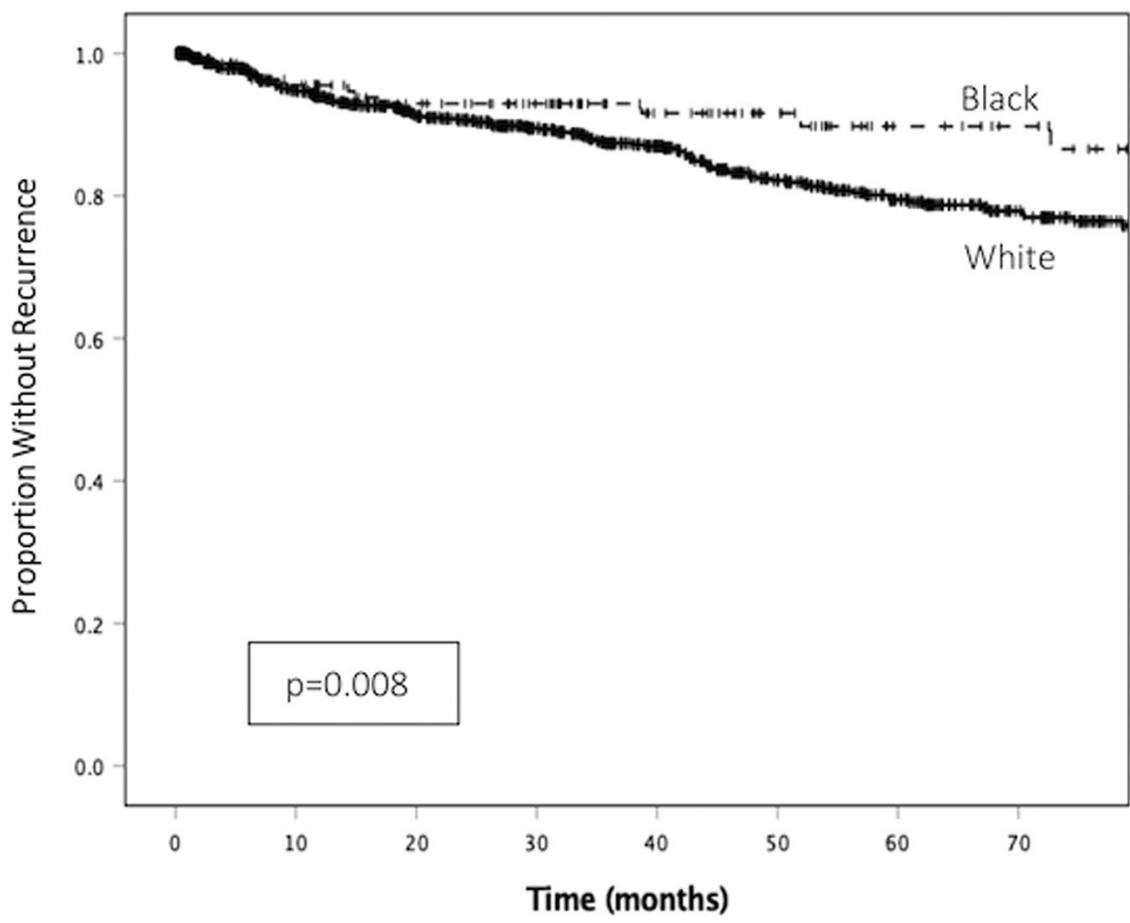

FIGURE 1 Recurrence-free survival by race 


\section{(A) Small Bowel Neuroendocrine Tumors}
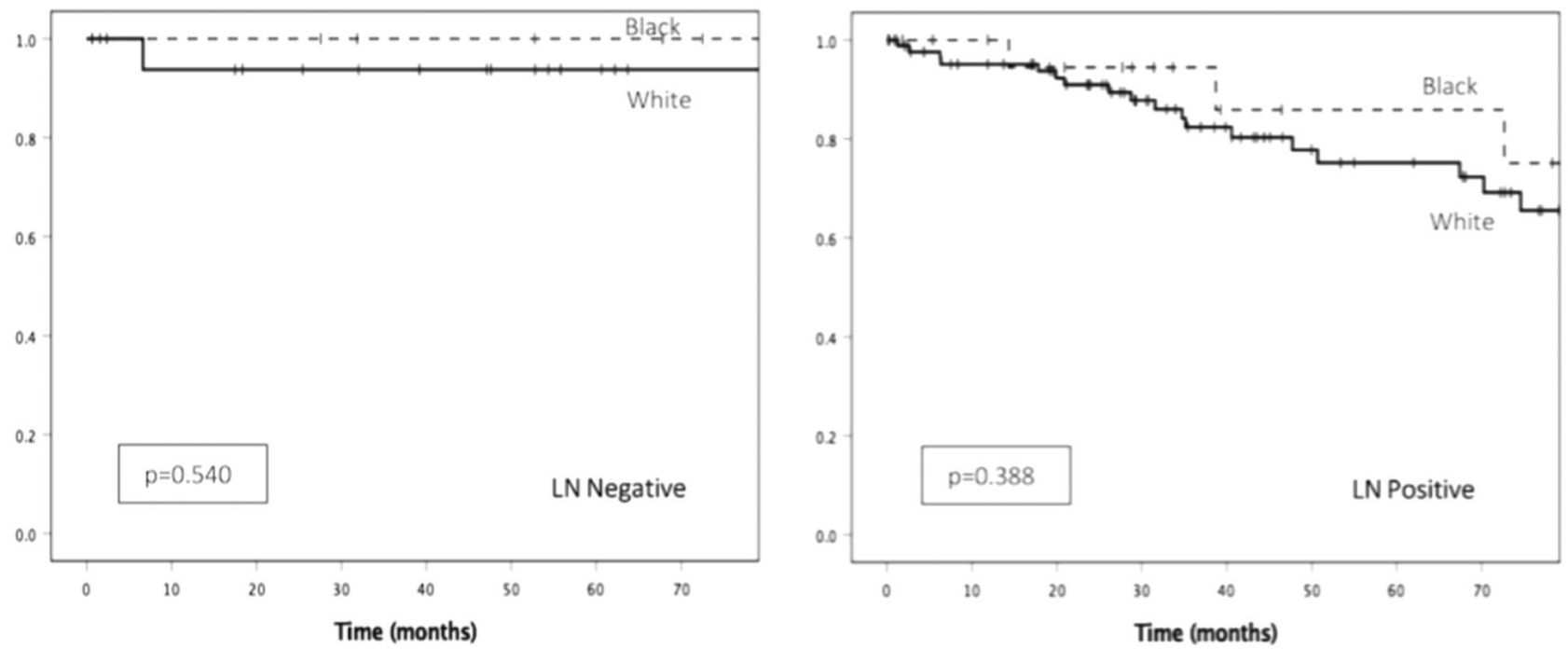

(B)

\section{Pancreas Neuroendocrine Tumors}
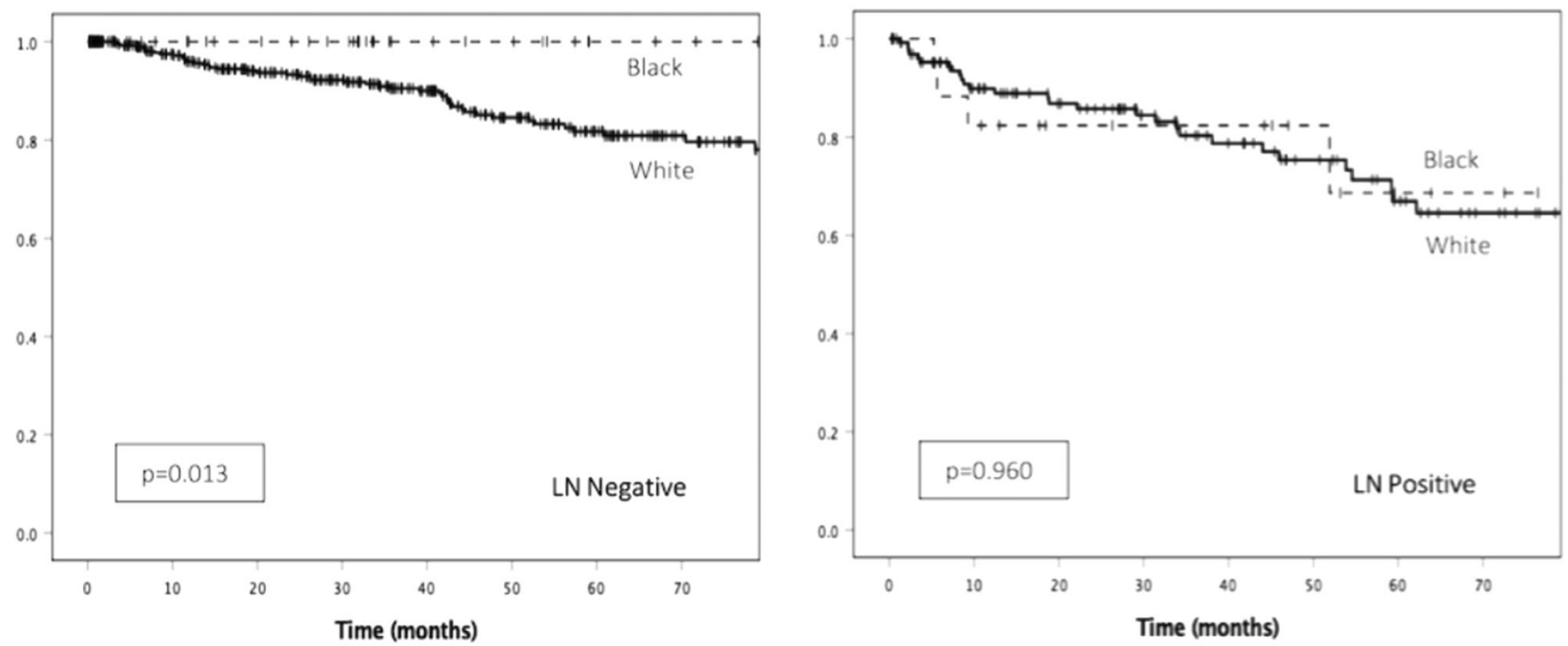

FIGURE 2 A, Small bowel NET lymph node positivity recurrence-free survival by race. B, Pancreatic NET lymph node positivity recurrence-free survival by race. NET, neuroendocrine tumor

and NCDB data. Zhou et $\mathrm{al}^{11}$ found that Black patients have a worse overall survival and pancreatic neuroendocrine tumor disease-specific survival, which was attributed primarily to advanced stage disease at diagnosis and decreased access to surgery. More specifically, they found that there was no difference in these outcomes for Black and White patients who did undergo surgery, which corroborates our findings. ${ }^{11}$ In another study, Fraenkel et al $^{13}$ reported that the incidence of small bowel neuroendocrine tumors was higher in Black patients compared with White patients, similar to our findings, although they found no difference in the incidence of pancreatic neuroendocrine tumors.

TABLE 3a Cox regression for recurrence-free survival: small bowel

\begin{tabular}{|c|c|c|c|c|}
\hline Variable & Univariate analysis & $P$ value & $\begin{array}{l}\text { Multivariable } \\
\text { analysis }\end{array}$ & $P$ value \\
\hline \multicolumn{5}{|l|}{ Race } \\
\hline Black & $0.397(0.118-1.335)$ & & $0.539(0.159-1.821)$ & \\
\hline $\begin{array}{l}\text { Lymph node } \\
\text { positivity }\end{array}$ & $5.040(0.678-37.48)$ & .114 & & \\
\hline
\end{tabular}


TABLE 3b Cox regression for recurrence-free survival: pancreas

\begin{tabular}{|llll} 
Variable & Univariate analysis & P value & \multicolumn{1}{c}{$\begin{array}{l}\text { Multivariable } \\
\text { analysis }\end{array}$} \\
\hline Race & & Reference \\
\hline White & Reference & .074 & $0.433(0.159-1.18)$ \\
\hline Black & $0.400(0.146-1.09)$ & & 103 \\
\hline Lymph node positivity & $1.97(1.28-3.03)$ & .002 & $1.99(1.30-3.06)$ \\
\hline
\end{tabular}

In this study, the improved RFS observed in Black patients may be attributed to the epidemiologic differences in the site of presentation of gastroenteropancreatic neuroendocrine tumors. Specifically, Black patients presented with a higher rate of small bowel neuroendocrine tumors than White patients. Small bowel neuroendocrine tumors generally have a better prognosis than pancreatic neuroendocrine tumors, which was present at higher rates in White patients in this study. ${ }^{9}$ While there was not a statistically significant difference in 5-year RFS seen in this cohort, this may be due to the relatively small sample size and number of events. The impact of lymph node positivity in neuroendocrine tumors also may not be uniform across all disease sites. Our group recently reported that there is limited prognostic significance of lymph node positivity in small bowel neuroendocrine tumors if fewer than 4 lymph nodes are positive. ${ }^{14}$ It is important to note that the average number of positive lymph nodes in this study was 3.4 and 3.8 in small bowel neuroendocrine tumors for Black and White race patients, respectively. Thus, the improved RFS observed in Black patients in the entire GEP cohort, despite a higher rate of lymph node-positive disease, may be reflective of the fact that the prevalence of small bowel neuroendocrine tumors seems to be higher in Black compared with White patients, where there is limited prognostic value of lymph node-positive disease. Finally, the improved survival in node negative Black patients with pancreatic neuroendocrine tumors is a relatively novel finding and will require more investigation as these numbers were small.

The generalizability of the results of this study are limited by the population, which exclusively comes from large, metropolitan, academic institutions. We were also only able to examine Black and White races due to the small sample size of other races in the database. The small sample size of some site-specific analyses may have limited the power of the analysis. As with any retrospective study, there are concerns for selection bias and difficulty capturing complete RFS data. Regardless, to our knowledge this represents the largest study evaluating the interaction of race with pathologic and oncologic outcomes of gastroenteropancreatic neuroendocrine tumors at centers that manage a high volume of this rare disease.

\section{5 | CONCLUSION}

In contrast to published literature, in this multi-institutional study, Black patients did not have inferior outcomes from neuroendocrine tumors, and in fact had improved RFS compared with White patients, despite presenting with more adverse pathologic characteristics. This improved RFS seen in Black patients may be attributed to the epidemiologic differences in the site of presentation of GEP neuroendocrine tumors and the variable prognostic value of lymph node-positive disease.

\section{GROUP/CONSORTIUM MEMBERS}

Zaheer Kanji MD², Eleftherios Makris $\mathrm{MD}^{3}$, Eliza Beal MD ${ }^{4}$, Roheena Panni $\mathrm{MD}^{5}$, Paula Marincola Smith $\mathrm{MD}^{6}$, Megan Beems MD ${ }^{7}$, Victoria Rendell $\mathrm{MD}^{8}$

1. Division of Surgical Oncology, Department of Surgery, Winship Cancer Institute, Emory University, Atlanta, Georgia

2. Department of Surgery, Virginia Mason Medical Center, Seattle, Washington

3. Department of Surgery, Stanford University Medical Center, Stanford, California

4. Division of Surgical Oncology, The Ohio State University Comprehensive Cancer Center, Columbus, Ohio

5. Department of Surgery, Washington University School of Medicine, St Louis, Missouri

6. Division of Surgical Oncology, Department of Surgery, Vanderbilt University Medical Center, Nashville, Tennessee

7. Division of Hepatopancreatobiliary and Advanced Gastrointestinal Surgery, Department of Surgery, University of Michigan, Ann Arbor, Michigan

8. Department of Surgery, University of Wisconsin School of Medicine and Public Health, Madison, Wisconsin.

\section{DATA ACCESSIBILITY}

The data that support the findings of this study are available from the corresponding author upon reasonable request.

\section{ORCID}

Rachel M. Lee (D) http://orcid.org/0000-0003-2704-839X

Maria C. Russell (D) http://orcid.org/0000-0003-1584-6992

\section{REFERENCES}

1. Hallet J, Law CHL, Cukier M, Saskin R, Liu N, Singh S. Exploring the rising incidence of neuroendocrine tumors: a population-based analysis of epidemiology, metastatic presentation, and outcomes. Cancer. 2015;121(4):589-597. 
2. Modlin IM, Oberg K, Chung DC, et al. Gastroenteropancreatic neuroendocrine tumours. Lancet Oncol. 2008;9(1):61-72.

3. Modlin IM, Kidd M, Latich I, Zikusoka MN, Shapiro MD. Current status of gastrointestinal carcinoids. Gastroenterology. 2005;128:1717-1751.

4. Pasricha G, Padhi P, Daboul N, Monga DK. Management of welldifferentiated gastroenteropancreatic neuroendocrine tumors (GEPNETs): a review. Clin Ther. 2017;39(11):2146-2157.

5. Dasari A, Shen C, Halperin D, et al. Trends in the incidence, prevalence, and survival outcomes in patients with neuroendocrine tumors in the United States. JAMA Oncol 2017;3(10):1335-1342.

6. Tsikitis VL, Wertheim BC, Guerrero MA. Trends of incidence and survival of gastrointestinal neuroendocrine tumors in the United States: a SEER analysis. J Cancer. 2012;3:292-302.

7. Fraenkel M, Kim MK, Faggiano A, Valk GD. Epidemiology of gastroenteropancreatic neuroendocrine tumours. Best Pract Res Clin Gastroenterol. 2012;26(3):691-703.

8. Oronsky B, Ma PC, Morgensztern D, Carter CA. Nothing but NET: a review of neuroendocrine tumors and carcinomas. Neoplasia. 2017;19(12):991-1002.

9. Hauso $\mathrm{O}$, Gustafsson $\mathrm{BI}$, Kidd $\mathrm{M}$, et al. Neuroendocrine tumor epidemiology. Cancer. 2008;113:2655-2664.

10. St Julien J, Huo J, Shih YCT, et al. Impact of race on surgical management of pancreatic neuroendocrine tumors. J Clin Oncol. 2016;34(15):e18081-e18081.
11. Zhou H, Zhang $\mathrm{Y}$, Wei $\mathrm{X}$, et al. Racial disparities in pancreatic neuroendocrine tumors survival: a SEER study. Cancer Med. 2017;6(11):2745-2756.

12. Liu JH, Zingmond DS, McGory ML, et al. Disparities in the utilization of high-volume hospitals for complex surgery. JAMA. 2006;296(16):1973-1980.

13. Fraenkel M, Kim M, Faggiano A, de Herder W, Valk G. Incidence of gastroenteropancreatic neuroendocrine tumours: a systematic review of the literature. Endocr Relat Cancer. 2014;21(3):R153R163.

14. Zaidi MY, Lopez-Aguiar AG, Dillhoff M, et al. Prognostic role of lymph node positivity and number of lymph nodes needed for accurately staging small bowel neuroendocrine tumors. JAMA Surg. 2019;154(2):134-140.

How to cite this article: DePalo DK, Lee RM, Lopez-Aguiar

$A G$, et al. Interaction of race and pathology for neuroendocrine tumors: Epidemiology, natural history, or racial disparity? J Surg Oncol. 2019;120:919-925.

https://doi.org/10.1002/jso.25662 